\title{
US Navy superconductivity programme
}

\author{
DONALD U GUBSER \\ Naval Research Laboratory, Washington DC 20375-5000. USA
}

The U.S. Navy has had a long-running and sustained programme dating back to 1946 supporting superconductivity and its technology. This is due to a recognition of the enormous military benefits that could be achieved if the unique properties of superconducting materials could be trapped in field-operating systems. The present Navy's programme is broadly based with an emphasis on field-operational systems. The Navy is committed to developing a science and technology base for superconducting materials, both high-temperature superconductors (HTS) and lowtemperature superconductors (LTS), and to undertaking key system tests leading to the eventual implementation of the technology. The programme is well integrated throughout the Navy with four organizations leading the way in specific areas. Those organizations are (i) the Office of Naval Research (ONR) and the Office of Naval Technology (ONT) [responsible for contract funding in all areas of Naval interest], (ii) the Naval Research Laboratory (NRL) [responsible for fundamental property studies, materials research and development, and electronic and space applications], (iii) the Naval Coastal Systems Center (NCSC) [responsible for SQUID magnetometer systems development], and (iv) the David Taylor Research Centre (DTRC) [responsible for power applications of superconductivity and ship propulsion]. Other Navy organizations participate in the total programme and all are coordinated by the Naval Consortium for Superconductivity (NCS).

The Navy's superconductivity programme includes work in HTS and LTS and is divided into 5 main thrust areas. They are (i) fundamental properties and materials research [mostly HTS], (ii) SQUID magnetometers and gradiometers [mostly LTS], (iii) superconducting ship propulsion [exclusively LTS], (iv) electronic applications including digital circuitry and IR sensors [50\% LTS and 50\% HTS] and (v) space experiment [exclusively HTS]. About $\frac{2}{3}$ of a total Navy's budget of $\$ 21 \mathrm{M}$ is split equally between fundamental properties/material research and the space experiment. SQUID research accounts for about $\frac{1}{6}$ of the budget with the remaining going to power applications and electronic applications.

Almost half of the Navy's programme is focused on fundamental research and materials development. The Navy believes this is essential if the technology is to meet its potential for Naval systems. The programme is broadly based with major efforts in theory, materials synthesis (primarily films), $J_{c}$ and flux flow studies, surface resistance and noise studies, and radiation damage. The programme includes strong efforts at NRL and other inhouse laboratories, as well as contract programmes at universities and industries.

Superconducting electronics development in the Navy began around 1966. Today the programme is relatively broad-based working on IR sensors (HTS), millimeter and microwave devices (HTS and LTS), directional antennas (HTS), and digital circuit elements (LTS). Key to this programme is assessments of the impact of the technology 
on specific systems performance vis-a-vis competing technologies. This programme will develop, fabricate and test device elements to certain their performance advantages and limitations. Close ties with universities, industries, and other DoD efforts in superconducting electronics gives the programme an unusual breadth.

The space experiment at NRL began in 1988 and is designed to demonstrate the feasibility of millimeter wave and microwave electronics for space use. The programme includes material scientists, and electronic and spacecraft engineers. Various devices are being manufactured by over 15 different industries, made from a variety of HTS materials, and will be delivered to NRL by June 1990 for testing and evaluation. Devices which meet design criteria will be assembled into a spacecraft and launched into space sometime in the 1992 time frame. The tests are designed to demonstrate the virtues of superconducting electronics in space and to space-qualify the HTS devices. Space qualification means that the devices operate after the shock of space launch and exposure to space radiation environment. Future flights after 1992 are envisioned and will become more system-oriented, as the individual device performance warrants.

The SQUID detection/surveillance systems development at NCSC began in 1969 and has focused on field-operational demonstrations of the technology. Unanticipated problems associated with operation in motion while in the ambient earth's magnetic field had to be solved before the needed sensitivity levels could be reached. A gradiometer system designed to meet the requirements of Naval operations was built by Sperry Univac in 1985 and has been successfully demonstrated in field tests. This gradiometer is now being refurbished with newer sensors and electronics under a contract with IBM and will be used in subsequent tests. The original system as well as the refurbished system in based on bulk, niobium $\mathrm{Nb}$ technology. Advanced systems are now being designed which will use thin film technology, but still use $\mathrm{Nb}$ as the superconducting material. Thin film systems are expected to be smaller and less sensitive to certain types of system noise. Also under study is the eventual design of a SQUID system based on HTS materials. Important issues of magnetic and thermal noise, flux motion and hysteresis need to be improved before the HTS SQUID system becomes viable as a Navy, field operational unit.

Superconducting ship propulsion development at DTRC began in 1969. This programme has successfully demonstrated that industrially built $(3000 \mathrm{Hp})$ superconducting motors can be installed in a small Naval vessel and operated at sea. $\mathrm{NbTi}$ wire was used in the magnet construction. The Navy has been reluctant to take the next step to develop a larger scale demonstration on a mid-sized Naval ship because (i) a reluctance to convert to a radically new drive system, (ii) a reluctance to depend on a liquid helium technology, specifically in combat, and (iii) cost of a mid-sized Naval vessel demonstration would be large and particularly burdensome in times of budget austerity. Progress is being made, however, in that the Navy has decided to make a major commitment to eléctric drive wherein superconductivity is seen as an advanced, but evolutionary concept. With this plan in mind, the Navy has committed funds now to explore magnet stability for forces, strains and vibrations anticipated in a Naval systems. The programme will also explore advanced LTS conductor design (aluminum-stabilized matrix, and $\mathrm{Nb}_{3} \mathrm{Sn}$ conductors) as well as improved refrigeration designs and tests. Only LTS materials are considered in this programme at the present time. 
Navy scientists and managers are actively involved with other major DoD efforts in superconductivity. They serve as reviewers, advisors and in some cases as agents in a number of programmes. In this manner the Navy's programme is thoroughly integrated into the overall efforts of the nation as a whole and committed to the rapid development of superconducting products, military or commercial. 